\title{
Beyond the Knife: The Evolving Nonsurgical Management of Oligometastatic Colorectal Cancer
}

\author{
Sharlene Gill, MD, MPH, MBA, FRCPC, David M. Liu, MD, FSIR, FRCPC, Harshani M. Green, MBBS, MRCP, \\ and Ricky A. Sharma, MA, MBA, Bchir, FRCP, FRCR, PhD
}

\section{OVERVIEW}

In patients with liver-limited oligometastatic disease, the goal of treatment can be curative intent. Historically, this was accomplished in patients presenting with upfront resectable disease. The availability of increasingly efficacious chemotherapy and biologic combinations with encouraging response rates led to the potential to convert unresectable disease to resectability. Beyond the backbone of surgery, we now have a portfolio of locoregional strategies to consider.From an interventional radiology perspective, the use of portal vein embolization can facilitate hypertrophy of the liver in anticipation of resection, thus converting unresectable disease to one amenable to a surgical approach with curative intent. Technological advances in liver-directed ablative therapies have afforded the possibility of eliminate radiographically evident disease with the hope for long-term disease control. Advanced radiotherapy techniques are further increasing the therapeutic options for patients with metastatic colorectal cancer. Improvements in external-beam radiotherapy over the past 2 decades include image-guided radiotherapy, intensity-modulated radiotherapy, stereotactic body radiotherapy, and proton-beam therapy. Finally, selective internal radiation therapy (SIRT) with microspheres labeled with the $\beta$-emitter ${ }^{90} Y$ enable targeted delivery of radiation to hepatic tumors. A coordinated multidisciplinary approach is required to integrate these nonsurgical adjuncts in an evidence-based manner to optimize outcomes for patients with potentially resectable metastatic disease. In this article, we summarize recent developments in systemic therapy, radiotherapy, and interventional liver-directed therapies that have changed the treatment landscape for patients with oligometastatic colorectal cancer.

A n estimated 140,250 Americans will be diagnosed with colorectal cancer (CRC) in $2018 .^{1}$ Of these, approximately $20 \%$ will present with de novo metastatic disease, and among those presenting with earlier-stage disease treated with resection, another $35 \%$ will relapse with distant metastatic disease after an initial disease-free interval. For the estimated $30 \%$ to $5 \%$ of patients with liver-limited or oligometastatic disease, it has long been recognized that surgical metastasectomy may achieve durable disease control and potentially cure.

However, less than $10 \%$ of patients with metastatic CRC $(\mathrm{mCRC})$ present with upfront resectable disease. ${ }^{2}$ Consequently, to achieve the goal of converting potentially resectable metastatic disease to resectability requires a multidisciplinary approach, including medical and surgical oncologists and, increasingly, radiation oncologists and interventional radiologists. Herein, we summarize recent developments in systemic therapy, radiotherapy and interventional liver-directed therapies that have changed the treatment landscape for patients with oligometastatic CRC.

\section{SYSTEMIC THERAPY FOR CONVERSION: WHEN RESPONSE RATE IS THE GOAL Choice of Chemotherapy Backbone}

The choice of chemotherapy regimen is dictated by several factors, including efficacy, tumor RAS status, toxicity, cost, and patient preference. Ultimately, however, when the intent is to convert potentially resectable disease to a complete RO resection, the primary metric of efficacy is typically response rate. For patients with good performance status, combination chemotherapy regimens with 5-fluorouracil and oxaliplatin (FOLFOX), irinotecan (FOLFIRI), or both (FOLFOXIRI) are all reasonable considerations. Because of the concern for irinotecan-related steatohepatitis, ${ }^{3}$ FOLFOX is commonly preferred; however, FOLFIRI remains an option, particularly in patients who have recently received FOLFOX in the adjuvant setting. Objective response rates of $45 \%$ to $55 \%$ were demonstrated with the doublet regimens in the prebiologic era. ${ }^{4,5}$ Trials comparing the triplet regimen of FOLFOXIRI to FOLFIRI demonstrated significantly higher response rates $(60 \%$ vs. $34 \%$; $p<$ .0001) with higher rates of secondary liver resection among patients with initially unresectable disease $(15 \% \text { vs. } 6 \%)^{6}$

\footnotetext{
From the BC Cancer-Vancouver and Vancouver General Hospital, University of British Columbia, Vancouver, BC, Canada; National Institute for Health Research University College London Hospitals Biomedical Research Centre, UCL Cancer Institute, University College, London, United Kingdom.

Disclosures of potential conflicts of interest provided by the authors are available with the online article at asco.org/edbook.

Corresponding author: Sharlene Gill, MD, MPH, MBA, FRCPC, BC Cancer-Vancouver, 600 West 10th Ave., Vancouver, BC V5Z 4E6, Canada; email: sgill@bccancer.bc.ca.
} 


\section{Role of Biologics}

With respect to biologics, the benefit of the VEGF monoclonal antibody bevacizumab in potentially resectable disease, with a goal of maximizing response rates, is still uncertain. In randomized trials, the addition of bevacizumab to oxaliplatincontaining chemotherapy did not meaningfully improve response rates, ${ }^{7}$ whereas a modest response rate improve was observed with irinotecan and 5-fluorouracil in combination with bevacizumab when administered as the IFL regimen. ${ }^{8}$ In the context of triplet therapy, the TRIBE trial of bevacizumab with FOLFOXIRI demonstrated a response rate of $65 \%$, similar to that previously reported with FOLFOXIRI alone. ${ }^{6}$ Beyond the questionable incremental improvement in response rates with bevacizumab, the concerns regarding operative toxicity with respect to bleeding, impaired wound healing, and impaired hepatic regeneration remain despite conflicting evidence. ${ }^{9,10}$ As a consequence, it is recommended that bevacizumab be withheld for 6 to 8 weeks before resection, recognizing the 3-week half-life of this agent.

The incremental improvement in response rates with EGFR monoclonal antibodies-cetuximab and panitumumab-is somewhat compelling in the $40 \%$ of patients with mCRC who have RAS wild-type (WT) disease; however, this is also not without controversy. The phase II CELIM trial of cetuximab with FOLFOX or FOLFIRI demonstrated response rates of $57 \%$ to $68 \%$ with a $38 \%$ RO resection rate. ${ }^{11}$ In another randomized trial from China, a response rate of $57 \%$ was reported with doublet chemotherapy plus cetuximab versus $29 \%$ with chemotherapy alone. ${ }^{12}$ However, the new EPOC trial of FOLFOX with or without cetuximab in the peri-metastasectomy setting showed a significantly detrimental impact on progressionfree survival with the addition of cetuximab (HR 1.48; $p=.030) \cdot{ }^{13}$ Although this study was in the upfront resectable setting, it raises concerns regarding the application of

\section{PRACTICALAPPLICATIONS}

- When the goal is resectability, the selection of a chemotherapeutic regimen is based upon what will permit the best response rates with the least toxicity.

- As hepatotoxicity is correlated with cumulative exposure, the duration of chemotherapy should be determined by when disease becomes resectable rather than treating to maximal response.

- Incorporation of an ablation strategy is an important component of a multispecialty treatment approach when considering eradication of colorectal liver metastases.

- Promising local control following stereotactic body radiotherapy for colorectal oligometastases has been demonstrated in several studies.

- A coordinated multidisciplinary approach is required to integrate all available nonsurgical modalities in an evidence-based manner, in order to optimize outcomes for patients with potentially resectable metastatic colorectal cancer. a cetuximab-containing regimen in patients who may be proceeding to a hepatic resection.

More recently, data on the influence of primary tumor location on response to EGFR-directed therapy has reignited interest in this biologic strategy in patients with left-sided RAS WT disease. In the randomized VOLVI trial of FOLFOXIRI with or without panitumumab, a response rate of $90 \%$ was observed in patients with left-sided RAS WT unresectable ( $\mathrm{mCRC}$ ), with a secondary resection rate of $60 \%$ among patients treated with the quadruplet regimen. ${ }^{14}$ Although this was a relatively smaller study with 96 selected well and younger patients, the response rates reported are very encouraging for the utility of an EGFR-containing regimen in appropriately selected patients.

Another important consideration is the duration of therapy in patients for whom resectability is the goal. Because best response is typically observed within the first six to eight cycles of treatment, if sufficient response to be considered for resection has not been achieved within 4 months of therapy, the likelihood of achieving resectability with continued therapy is low. Recognizing that treatment-related toxicity, particularly hepatotoxicity, is correlated with number of administered cycles, it is equally important to recognize that systemic therapy should continue to resectability and not necessarily to maximal response. Thus, close coordination between the medical oncologist and the surgical and interventional oncologists is imperative.

\section{INTERVENTIONAL RADIOLOGY: THE DRIVE TO SURGICAL RESECTION OR CURATIVE INTENT Portal Vein Embolization}

The liver demonstrates unique regenerative abilities because of its histopathologic and functional architecture. In brief, liver perfusion is based on inflow of hepatic arterial flow and portal venous flow. The organ itself is organized in parallel functional units (hepatic lobules) and demonstrates the ability to rapidly proliferate and regenerate in the setting of acute injury/insult. As a result of this condition, a large fraction of liver tissue may be resected in anticipation of hepatic regeneration. However, there are limitations to the compensation response. In liver with no compromise to hepatic reserve (no chemotherapy, noncirrhotic, no portal hypertension), up to $80 \%$ of the liver may be resected with extremely low incidence of hepatic failure. In the setting of compromise to hepatic reserve, up to $60 \%$ of the liver may be resected with a slightly higher incidence of hepatic failure.

In patients presenting with high-volume liver nonresectable metastatic disease, two options exist to facilitate conversion to surgical resectability: (1) tumor downsizing through systemic or locoregional therapy and (2) hypertrophy of the remaining liver. The decision to pursue these strategies in isolation or in combination largely depends on review in a multidisciplinary setting, drawing from the expertise of the medical oncologist, surgical oncologist, radiologist, and interventional radiologist. ${ }^{15}$

Techniques have been developed to facilitate hypertrophy of the liver in anticipation of surgical resection, thus converting 
potential nonresectability to curative intent. The use of portal vein embolization is predicated on knowledge that aggressive surgical ligation of the portal vein resulted in a clinically measurable compensator hypertrophic response in the remaining liver. Diversion of blood flow (causing increased hydrostatic pressure and transient portal hypertension), as well as increased expression of hepatotrophic mediators, has been theorized to facilitate the hypertrophic change in the residual liver, facilitating conversion of patients whose disease may not be surgically resectable to those with resectable disease. ${ }^{16}$

Various techniques have been described with respect to portal vein embolization, but the general principle is based on minimally invasive image-guided techniques. The general approach is summarized as follows: Percutaneous portal venous access is obtained through the liver under ultrasonographic or fluoroscopic guidance. Following this, selective catheterization of the individual branches of the portal vein within the liver volume that is intended for surgical resection is selectively embolized with particles, coils, liquid embolics (glue), or combinations of such, to divert portal venous flow into the portion of liver that is intended to remain, as well as increased expression of hyperproliferation factors secondary to the insult to the portion of liver that is intended for surgical resection.

Follow-up CT, typically 6 weeks, is performed to measure the degree of relative hypertrophy of the future liver remnant and overall total liver volume with anticipation of surgical resection if appropriate hypertrophy occurs. Hypertrophic changes then typically occur immediately and reach their nadir at 4 to 6 weeks, at which time reassessment for resectability based on the future liver remnant growth will be conducted. Changes in future liver remnant as high as $81 \% \pm 47 \%$ have been reported by using various embolic materials; ${ }^{17}$ the general consensus is that microspheres or cyanoacrylate results in increased growth. ${ }^{18}$ Postsurgical survival is reported to be equivalent or superior to that among those patients undergoing surgical resection alone (5-year survival, $44 \%$ vs. $35 \%$, with a statistically significant decrease in intrahepatic recurrences: $26 \%$ vs. $76 \%$; $p .01) .{ }^{19}$ This situation may be explained through the delay in surgery, providing a biologic test of time, or the facilitation of more aggressive surgical resection.

In summary, portal vein embolization is intended to extend the resection margins and optimization of the function of the remaining remnant, lowering the potential for liver failure. Aggressive and creative approaches can be developed to remove all visible disease through this adjunct procedure, but they require thoughtful review of the imaging by both surgeon and interventional radiologist in the setting of a multidisciplinary team. ${ }^{15,18}$

\section{Ablation Strategies}

The prognosis in patients with colorectal liver metastasis is largely determined by the degree of liver tumor infiltration. The rationale and use of liver-directed therapies for the treatment of resectable disease, or potentially locally curative therapy through surgical resection, have been established with both long-term population-based outcomes and smaller randomized control trials despite high rates of recurrence within the liver. ${ }^{20,21}$

Various energy-based ablative platforms are available (cryoablation, microwave ablation, irreversible electroporation), with most of the published literature relating to radiofrequency ablation. A discussion relating to the technology platforms is beyond the scope of this article, and interested readers may refer to review articles specific to the topic. Clinically, radiofrequency ablation is widely considered the most mature in terms of both technology and publication, and it remains the standard by which new ablative therapies (including stereotactic radiotherapy [SBRT]) are measured. ${ }^{22}$

Although the acceptance of hepatic resection and conversion to resectability have become mainstream, discussion and research on curative intent ablative therapies largely entail single-arm studies that often are retrospective. Furthermore, the high rate of recurrence of liver metastasis in patients undergoing curative intent questions the utility of aggressive surgical resection in the setting of small tumor burden. Because of the high variability in patient selection, randomized controlled trials comparing surgical resection to curative intent ablation have been limited. ${ }^{23}$

As a result of the variations in morphology, technique, and technologies, 5-year survival benefit has been used as a primary measure of efficacy, based on the low rates seen (145-year survival rate of $14 \%$ in patients with distant spread of disease, most hepatic) with optimized chemotherapeutic and biologic regimens. ${ }^{24}$

Many articles have reported outcomes with the incorporation of radiofrequency ablation into clinical practice. However, the variability of application (e.g., oligometastatic disease with curative intent, extension of irradiation of tumors that cannot be resected as an adjunct to resection, treatment upon recurrence, neoadjuvant setting) result in high variability in patient selection. Table 1 outlines the more commonly cited literature. ${ }^{25-35}$

Two studies (by Kim and colleagues ${ }^{36}$ and Ruers and colleagues ${ }^{30}$ ) provide some perspective on the indications and efficacy of ablation in the setting of colorectal liver metastasis. Kim and colleagues retrospectively reported on a subset of patients presenting with solitary colorectal liver metastasis of less than $3 \mathrm{~cm}$, comparing surgical resection to radiofrequency ablation with curative intent. In the subset of patients who met these criteria, no significant difference was observed with respect to disease-free survival and overall survival ( $p=.962$ ) between patients undergoing surgical resection and those receiving radiofrequency ablation. ${ }^{36}$

Ruers and colleagues' recent publication of the CLOCC data set (EORTC Intergroup phase II study [EORTC 40004]), examining a strategy of colorectal liver metastasis eradication through a surgical resection/ablation approach, had demonstrated a significant improvement in all clinically relevant parameters, highlighted by an 8-year overall survival rate of $56.9 \%$ versus $8.9 \%$ ( $\mathrm{HR} 0.58 ; 95 \% \mathrm{Cl}, 0.38-0.88$; $\mathrm{p}<.01$ ) comparing radiofrequency ablation plus chemotherapy 
versus chemotherapy alone. ${ }^{30}$ One may infer from this landmark study that curative-intent locoregional therapies result in improvement in survival and greater control of progression compared with systemic therapy alone. ${ }^{30}$

With these two studies, two emerging roles for ablation become apparent: (1) in patients with low-volume disease amenable to curative ablation as opposed to surgical resection due to the expected high rates of recurrence and (2) in patients presenting with a high burden of disease that is nonresectable, even with conversion therapy that may involve ablation only, or as a combinatorial approach to eradication of all identifiable tumor burden.

The technical limitations of radiofrequency ablation in lesions less than $3 \mathrm{~cm}$ (near vascular structures, subcapsular, central) may be overcome by microwave ablation, cryoablation and irreversible electroporation. However, controversy remains as to whether patients presenting with lesions less than 3-cm benefit from ablation, despite newer technologies allowing for larger ablation zones, as the biology of patients with large tumors (not necessarily large burden of disease) has not been clearly established. ${ }^{37}$

In summary, incorporation of an ablation strategy is an essential component of multispecialty care in the patient diagnosed with colorectal liver metastasis. The technologies and techniques have advanced substantially, with a greater emphasis on larger ablations and efficiency predicated on the justifiable assumption that removal of radiographically visible disease translates into a survival benefit.

\section{Embolization}

The fundamental concept of hepatic arterial embolic therapy is based on the process of tumor angiogenesis and the exploitation of the vascular capacitance of the tumor to administer high concentrations of chemotherapy loaded on calibrated microparticles through a process of ionic binding. Delivery of the chemotherapy is based on plasma ion exchange as a function of time, resulting in sustained concentrated locoregional delivery of the chemotherapeutic agent. Through the compartmentalization of chemotherapy, decreased toxicities, increased concentrations, and potentially improved tumor response may occur, in hopes of altering or regulating tumor biology, translating into an improvement in survival. Most commonly, irinotecan is used as the systemic therapeutic agent in the treatment of $\mathrm{mCRC}$, so termed DEBIRI. ${ }^{38}$

The current body of literature relating to the use of DEBIRI has been limited by risk of bias and the strength of reported results. A comprehensive critical review of the use of this technique, including elution profile of the various microsphere manufacturers, particle size, and chemotherapeutic

\section{TABLE 1. Summary of Radiofrequency Ablation Studies for Treatment of Metastatic Disease}

\begin{tabular}{|c|c|c|c|c|c|c|c|}
\hline Study (Year) & $\begin{array}{l}\text { No. of } \\
\text { Patients }\end{array}$ & Approach & $\begin{array}{l}\text { Median/Range No. } \\
\text { of Tumors }\end{array}$ & $\begin{array}{l}\text { Mean/Median } \\
\text { Diameter, cm }\end{array}$ & $\begin{array}{l}\text { Extrahepatic } \\
\text { Disease, \% }\end{array}$ & $\begin{array}{l}\text { 5-Year } \\
\text { Survival, \% }\end{array}$ & Comment \\
\hline Ruers et al $(2017)^{30}$ & 119 & $\begin{array}{l}\text { Percutaneous } \\
\text { laparoscopic }\end{array}$ & $4(1-9)$ & $<4 \mathrm{~cm}(\mathrm{NR})$ & 0 & 43.1 & $\begin{array}{c}\text { 8-year survival: } \\
\text { 39.5\%; } \text { RCT }^{*}\end{array}$ \\
\hline \multirow[t]{2}{*}{ Imai et al $(2017)^{33}$} & \multirow[t]{2}{*}{31} & \multirow{2}{*}{$\begin{array}{l}\text { Hepatect (Paviour) } \\
\quad+\text { RFA }\end{array}$} & 5 (2-25; total) & 29 (7-90; total) & \multirow[t]{2}{*}{0} & \multirow[t]{2}{*}{57} & \\
\hline & & & $2(1-4 ;$ RFA) & $1.3(0.4-3.0 ;$ RFA $)$ & & & \\
\hline $\begin{array}{l}\text { Agcaoglu et al } \\
(2013)^{32}\end{array}$ & 395 & Laparoscopic & $3(1-11)$ & 3.4 & 8 & 17 & $\begin{array}{l}\text { Extension of } \\
\text { RFA during } \\
\text { resection }\end{array}$ \\
\hline Bale et al $(2012)^{25}$ & 63 & Percutaneous & $2(1-14)$ & $2(0.5-13)$ & 0 & 27 & \\
\hline $\begin{array}{l}\text { Gillams et al } \\
\qquad(2009)^{26}\end{array}$ & 123 & Percutaneous & $2.1(1-5)$ & $2.9(0.9-5)$ & 0 & 24 & \\
\hline $\begin{array}{l}\text { Hamada et al } \\
\qquad(2012)^{27}\end{array}$ & 101 & Percutaneous & 1.7 & $2.3(0.5-9)$ & 27 & 21 & \\
\hline Kim et al $(2011)^{34}$ & 595 & $\begin{array}{l}\text { Open } \\
\text { percutaneous }\end{array}$ & 1.6 & $2.1(0.5-6.2)$ & 0 & $51(<3 \mathrm{~cm})$ & \\
\hline $\begin{array}{c}\text { Machi et al } \\
(2006)^{29}\end{array}$ & 100 & $\begin{array}{l}\text { Open } \\
\text { percutaneous } \\
\text { laparoscopic }\end{array}$ & 3.5 & 3 & 20 & 31 & \\
\hline $\begin{array}{l}\text { Van Tillborg et al } \\
\qquad(2011)^{35}\end{array}$ & 38 & $\begin{array}{l}\text { Open } \\
\text { percutaneous }\end{array}$ & 2.4 & $2.4(0.2-8.3)$ & $-^{* *}$ & 36 & $\begin{array}{l}\text { 8-year survival: } \\
24 \%\end{array}$ \\
\hline $\begin{array}{l}\text { Hammill et al } \\
\qquad(2011)^{28}\end{array}$ & 101 & Laparoscopic & 2.6 & $3-4$ & & $18-49^{+}$ & \\
\hline $\begin{array}{l}\text { Solbiati et al } \\
\qquad(2012)^{31}\end{array}$ & 99 & Percutaneous & 2 & $2.2(0.8-4)$ & 7 & 48 & $\begin{array}{l}\text { 7-year survival: } \\
25 \%\end{array}$ \\
\hline
\end{tabular}

*The only prospective randomized controlled trial to date.

**Extrahepatic spread with intent for surgical resection.

†Forty-nine percent for resectable disease with curative intent.

Abbreviations: NR, not reported; RCT, randomized, controlled trial; RFA, radiofrequency ablation.

Randomized controlled trial: 119 patients with confirmed unresectable disease by conventional anatomic approach randomly assigned to systemic therapy (59 patients) alone or to systemic therapy plus ablation/resection (51 patients). 
intensity, concluded that the small body evidence (primarily restricted to small retrospective analyses, and phase lla observational prospective studies) limited broad adoption of DEBIRI. ${ }^{38}$

Recently, Martin and colleagues reported on the largest randomized controlled trial to date of patients undergoing DEBIRI. ${ }^{39}$ They recruited 70 patients into this two-group study to compare FOLFOX6 and bevacizumab versus mFOLFOX6 and DEBIRI with or without bevacizumab in the first-line setting. The primary endpoint was response rate. The overall response rate was significantly greater in the interventional arm at 2 months (70\% versus 54\%; $p=.02$ ) and at 6 months (76\% versus $60 \% ; p=.05$ ), achieving success in the primary endpoint. $^{39}$

However, critics have stated concern regarding the introduction of additional chemotherapeutic agents in the experimental arm, questioning whether the reported improvement in outcome may be confounded by the systemic effects rather than the liver-directed therapy. Under these pretenses, a proper study would be best compared with a FOLFOXIRI/FOLFIRINOX regimen as opposed to what is being described in the study, which is FOLFOX alone. The authors concluded that the simultaneous administration of microspheres is safe and does not cause treatment delays or increase systemic toxicity of chemotherapy, with the added benefit of improved overall response rate, improved hepatic progression-free survival, and more durable overall progression-free survival (with a statistically increased incidence of downstage to surgical resection). Given the limited evidence, the use of DEBIRI should be reserved for further study.

\section{RADIOTHERAPY FOR OLIGOMETASTASES: ADVANCES IN EXTERNAL BEAM AND SELECTIVE-INTERNAL RADIATION}

Modern advanced radiotherapy techniques are increasing the therapeutic options for patients with oligometastatic mCRC. Improvements in external-beam radiotherapy (EBRT) over the past 2 decades include image-guided radiotherapy, intensity-modulated radiotherapy, SBRT, and proton-beam therapy.

Although the standard dose for primary rectal cancer is generally 45 to 54 Gy in 1.8-Gy fractions daily, some investigators report that hepatic metastases from CRC are more radioresistant than other liver metastases clinically. ${ }^{40,41}$ SBRT can potentially allow delivery of higher biologic doses of radiotherapy than conventional fractionation in three to five fractions over 2 weeks, with greater convenience for patients and potentially higher efficacy than conventional radiotherapy. It requires very accurate image guidance and motion management to ensure a high degree of spatial accuracy. At most centers, SBRT is generally offered to patients with up to three to five liver metastases and small treatment volumes ( $<8 \mathrm{~cm}$ lesion diameter), but it can be used to treat more lesions depending on their locations within the liver and larger tumors as long as sufficient normal functioning liver (700 mL minimum) can be spared. ${ }^{42,43}$ Larger lesions are commonly treated with three-dimensional conformal radiotherapy or intensity-modulated radiotherapy with 45 to 66 Gy in 1.8-Gy fractions. ${ }^{44}$

For all forms of external-beam radiotherapy to the liver, it is recommended that patients have high-quality triphasic CT planning scans, ideally with coregistration of MRI, with or without positron-emission tomography for accurate target delineation. ${ }^{42,43}$ Because the liver moves with breathing, techniques for motion management are essential, as is image-guided radiotherapy, such as cone-beam CT with or without radio-opaque fiducial markers. ${ }^{45,46}$ Techniques used by liver centers include abdominal compression, measured breath-hold, and gated-radiotherapy treatment during the breathing cycle. ${ }^{45,46}$ If four-dimensional CT is used, an internal target volume is defined to encompass the target volume in all respiratory phases. ${ }^{43}$

Many studies have evaluated the safety, feasibility, and toxicity profile of SBRT in the management of hepatic tumors. ${ }^{36,47-50}$ Particular caution must be exercised regarding the proximity of adjacent luminal gastrointestinal structures because all forms of EBRT can cause hemorrhagic gastritis with doses greater than $7 \mathrm{~Gy}$ to one third of the stomach ${ }^{51}$ or colonic/duodenal ulceration with doses greater than 30 Gy in three fractions. ${ }^{52}$ Departmental protocols are based on international guidance ${ }^{53}$; if substantial compromise of the planning target volume is likely to be required, the treating oncologist may decide to change to conventional fractionation.

Radiation-induced liver disease (RILD) has been the main limiting factor for the use of EBRT in hepatic tumors. RILD is characterized by central sinusoid congestion with adjacent hepatic atrophy ${ }^{54}$ and usually occurs from a few weeks to 4 months after irradiation. Traditionally, a 5\% risk for RILD after 32 Gy to the whole liver at 2 Gy per fraction is quoted. ${ }^{55}$ The risk for RILD is considered proportional to the mean dose of radiation to the normal tissue as the liver follows the parallel architecture model of radiobiology. ${ }^{43}$ By sparing sufficient functioning liver, doses up to $90 \mathrm{~Gy}$ have been reported to be safely given to up to a third of the liver volume. ${ }^{42,56,57}$ From the patient's perspective, nausea, elevated liver enzymes, acute skin erythema, asthenia, thrombocytopenia, and chest wall pain have been observed following EBRT.

Various studies have shown promising local control rates after SBRT as a focal treatment of CRC oligometastases in multiple organs, including liver, lung, and lymph nodes. . $^{5258-60}$ Some studies have included hepatic oligometastases from multiple primaries, including CRC.43,47,61-69 Studies that included only inoperable hepatic oligometastases from CRC are listed in Table 2. ${ }^{36,48-50,70,71}$ of note, rates of Common Terminology Criteria for Adverse Events (CTCAE) adverse events of grade 3 or greater toxicity range between $0 \%$ and $15 \%$ in the six studies listed in Table 1, confirming that SBRT is generally well tolerated.

Local control rates at 1 year for SBRT from three prospective and three retrospective studies of treatment for CRC hepatic metastases range between $50 \%$ and $100 \%$, and overall 


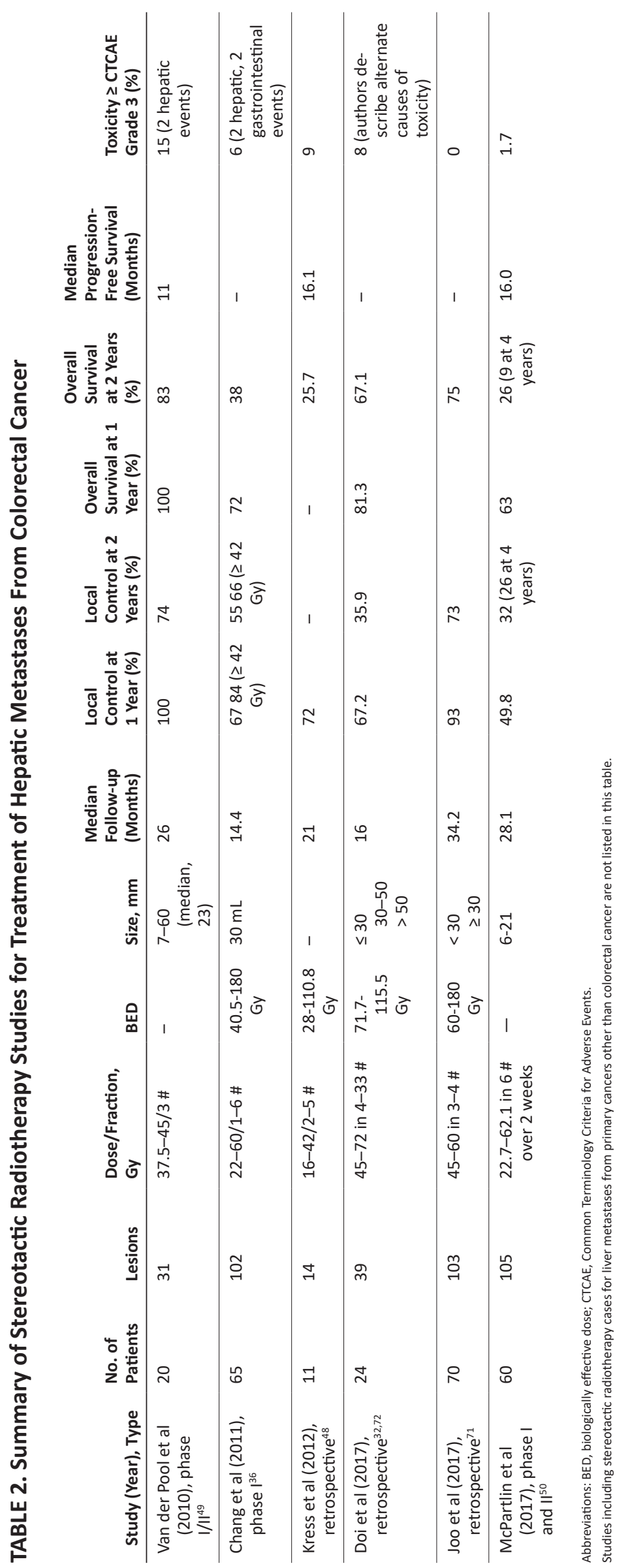

2142018 ASCO EDUCATIONAL BOOK | asco.org/edbook 
survival rates at 2 years range between $26 \%$ and $83 \%{ }^{36,48-50,70,71}$ However, out-of-field recurrences are common, with rates reported between $59 \%$ and $68 \% .^{36,50,71}$ Patient selection is therefore important; preferably, patients should be selected for SBRT if they seem less likely to develop other metastases in the near future, without or with the addition of systemic therapy. Clinical trials exploring this approach include those combining SBRT for mCRC with immunotherapy. ${ }^{73}$

A pooled analysis of 47 patients evaluated a range of doses between 22 and $60 \mathrm{~Gy}$ and showed that total dose, dose per fraction, and biologically effective dose (BED) correlated with local control $(p=.06) .{ }^{36}$ The best-fit curve estimated that a dose of $48 \mathrm{~Gy}$ or greater in three fractions (BED, 117 Gy) was required for a 1-year local control rate of greater than $90 \%$. In a separate study, a retrospective analysis of 70 patients showed that more prolonged local control was observed if higher doses were used; the optimal BED dose was greater than $132 \mathrm{~Gy} .{ }^{71}$ The 2-year local control rate for lesions receiving BED at greater than 132 Gy was 89\%; for those receiving 100 to $112 \mathrm{~Gy}$ and 60 to $80 \mathrm{~Gy}$, the rates were $83 \%$ and $52 \%$, respectively. ${ }^{71}$ The optimal dosimetric parameters for an effective ablative dose with minimal toxicity require further evaluation through clinical trials.

Proton-beam therapy uses charged particles to deposit dose at a defined depth with a very sharp fall-off in dose, potentially reducing irradiation of the surrounding normal tissue. One prospective phase II study of 89 patients with hepatic metastases, of varying histologic types (34 CRCs), used protons to doses of 30 to 50 gray equivalents in five fractions (BED, 48-100 Gy; relative biologic effectiveness, 1.1) and had a median follow up of 30.1 months. ${ }^{74}$ There were no CTCAE grade 3 or greater toxicities, and 1- and 3-year local control rates were $71.9 \%$ and $61.2 \%$, respectively. Interestingly, as suggested by other investigators using SBRT with photons, ${ }^{41} \mathrm{CRC}$ metastases in the photon-beam therapy study had worse local control than did other tumors. ${ }^{74}$ Somatic analyses showed that tumors with mutant KRAS and TP53 tumors were more radioresistant than tumors wild-type for the same genes, and that mutation of the KRAS oncogene was a strong predictor of poor local control. This study is an important demonstration that proton-beam therapy safe and effective to treat oligometastases from $\mathrm{mCRC}$.

\section{EBRT for Lung or Lymph Node Metastasis}

Lung is a common site for $\mathrm{MCRC}$, and SBRT is considered a treatment option for oligometastatic pulmonary metastases. The dose and fractionation depend on tumor size and proximity to areas at risk, including the chest wall, heart, and main bronchus. In one study, 60 patients were treated with doses of 48 to $60 \mathrm{~Gy}$ in three to four fractions, demonstrating 2-year local control rates of $80 \%$, with a median time to lung progression of 7 months. ${ }^{59}$ In another study, 44 patients with 69 CRC pulmonary metastases were treated with SBRT; ${ }^{75}$ 2-year overall survival rate was $67.7 \%$, and 2 -year local progression-free survival rate was $60.2 \%$. No CTCAE grade 3 or greater toxicities were reported.
In practice, SBRT is usually the second choice if surgical resection is not feasible. One study evaluated the outcomes of patients with pulmonary oligometastases after SBRT with those after pulmonary metastasectomy. ${ }^{76}$ Despite the selection bias (SBRT was second choice in this study if resection was not possible), 2-year local control rates did not significantly differ between the two study groups: $94 \%$ for SBRT and $90 \%$ for resection. ${ }^{76}$

Despite its widespread use at SBRT centers, there are limited high-quality data on the efficacy of SBRT for oligometastatic lymph node metastases. Because lymph node resection is not practiced widely for metastatic disease and can be technically challenging and morbid, SBRT is often the preferred treatment option for these patients and can offer them periods free from the toxicities of systemic therapy. In a retrospective study of 18 patients treated with SBRT for lymph node oligometastases from various primary tumors (seven CRC), a 1-year local control rate of $94 \%$ was observed and 1-year overall survival rate was $89 \%$. No CTCAE grade 3 or greater toxicities were observed. ${ }^{77}$ In general, doses ranging from 36 to $51 \mathrm{~Gy}$ in three to five fractions have been used; careful recording of acute and late toxicities is advised to ensure adequate clinical governance.

\section{Selective Internal Radiotherapy for Liver Metastasis}

Selective internal radiation therapy (SIRT) with resin or glass microspheres labeled with the $\beta$-emitter ${ }^{90} \mathrm{Y}$ enables targeted delivery of radiation to inoperable liver metastases from CRC. ${ }^{78-80}$ Whereas SBRT delivers high doses of external radiation in fractions, SIRT delivers a continuous dose of radiation inside the liver. The physical half-life of ${ }^{90} \mathrm{Y}$ is 64 hours, so patients must be aware of some simple radiation protection precautions for up to 7 days after SIRT. ${ }^{79}$ The treatment involves a workup procedure during which hepatic angiography is performed, aberrant vessels from liver to other organs are embolized with coils, and ${ }^{99 \mathrm{~m} T c-l a b e l e d ~ m a c r o-a g g r e g a t e d}$ albumin is injected as a surrogate of the treatment. ${ }^{78-80}$ The definitive treatment procedure is performed within 2 to 3 weeks of the workup procedure, during which millions of ${ }^{90} \mathrm{Y}$ microspheres are injected directly into the hepatic arterial vasculature. Because the ${ }^{90} \mathrm{Y}$-loaded microspheres preferentially localize in tumor arterial vasculature, very high radiation doses are delivered to tumors while tolerable radiation doses to normal hepatic tissue are maintained.

In $\mathrm{MCRC}$, SIRT is predominantly used as salvage therapy in treatment-refractory disease ${ }^{81-83}$ or in the second-line settings in combination with chemotherapy. ${ }^{79,84}$ Hendlisz and colleagues randomly assigned 46 patients with $\mathrm{mCRC}$ refractory to standard chemotherapy to protracted intravenous infusional 5-fluoroucil or the same chemotherapy (dose reduced) plus SIRT; they observed an improvement in median time to tumor progression of 2.4 months in favor of the combination treatment. ${ }^{83}$ Because of crossover to SIRT treatment from the control arm, overall survival could not be studied meaningfully. On the basis of this randomized controlled trial and published cohort studies, SIRT is widely reimbursed in high-income countries for the treatment of 
$\mathrm{mCRC}$ refractory to chemotherapy. There exists recently published guidance on the optimal approach to managing some of the most important complications of SIRT. ${ }^{85}$

The combination of SIRT with first-line chemotherapy is not widely used in clinical practice. The feasibility of combining SIRT with first-line FOLFOX chemotherapy as a combined radiosensitization approach for $\mathrm{mCRC}$ had previously been demonstrated by Sharma and colleagues in 2007. ${ }^{86}$ The FOXFIRE-SIRFLOX-FOXFIRE Global combined analysis of 1,103 patients assessed the efficacy and safety of SIRT with ${ }^{90} \mathrm{Y}$ resin microspheres when combined with first-line FOLFOX chemotherapy versus FOLFOX alone in patients with liver-only or liver-dominant $\mathrm{mCRC}$ (bevacizumab or other biologically targeted agent was allowed at the discretion of the investigators). This combined analysis demonstrated that despite improving objective response rate and liverspecific progression, the addition of SIRT did not affect overall survival and increased the frequency of grade 3 to 5 adverse events. ${ }^{87}$ The median improvement in local control of liver metastases exceeded 8 months, ${ }^{84}$ but the amount of chemotherapy and other anticancer therapy received by patients in the combination arm was significantly lower than that in patients randomly assigned to the control arm. Because approximately $16 \%$ of patients in these prospective clinical trials proceeded to have surgical resection of liver metastases after SIRT, ${ }^{49}$ current data suggest that liver surgery is safe after SIRT, as suggested by a recently published surgical series. ${ }^{85}$

In summary, recent advancements in systemic therapy, external and internal radiotherapy, and interventional radiology have substantially expanded our therapeutic armamentarium. A coordinated multidisciplinary strategy is required to integrate these nonsurgical adjuncts in an evidence-based manner to optimize outcomes for patients with potentially resectable metastatic disease.

\section{ACKNOWLEDGMENT}

R. A. S. is supported by the National Institute for Health Research University College London Hospitals Biomedical Research Centre, Cancer Research UK (Grant A8971 CRUK/ 07/030), and research grants from Sirtex Medical and BTG plc. Author contributions: All authors made a substantial contribution to the conception of this article, acquisition of literature from the literature searches, and interpretation of published data. All authors drafted at least one section of the article and revised it critically for important intellectual content. All authors gave final approval of this version to be submitted for publication.

\section{References}

1. Siegel RL, Miller KD, Jemal A. Cancer statistics, 2018. CA Cancer J Clin. 2018;68:7-30.

2. Khatri VPPN, Petrelli NJ, Belghiti J. Extending the frontiers of surgical therapy for hepatic colorectal metastases: is there a limit? J Clin Oncol. 2005;23:8490-8499.

3. Vauthey JN, Pawlik TM, Ribero D, et al. Chemotherapy regimen predicts steatohepatitis and an increase in 90-day mortality after surgery for hepatic colorectal metastases. J Clin Oncol. 2006;24:20652072.

4. Tournigand CAT, Achille E, Llego G, et al. FOLFIRI followed by FOLFOX6 or the reverse sequence in advanced colorectal cancer: a randomized GERCOR study. J Clin Oncol. 2004;22:229-237.

5. Goldberg RM, Sargent DJ, Morton RF, et al. A randomized controlled trial of fluorouracil plus leucovorin, irinotecan, and oxaliplatin combinations in patients with previously untreated metastatic colorectal cancer. J Clin Oncol. 2004;22:23-30.

6. Falcone A, Ricci S, Brunetti I, et al. Phase III trial of infusional fluorouracil, leucovorin, oxaliplatin, and irinotecan (FOLFOXIRI) compared with infusional fluorouracil, leucovorin and irinotecan (FOLFIRI) as first-line treatment of metastatic colorectal cancer: the Gruppo Oncologico Nord Ovest. J Clin Oncol. 2007;25:1670-1676.

7. Saltz LBCS, Clarke S, Díaz-Rubio E, et al. Bevacizumab in combination with oxaliplatin-based chemotherapy as first-line therapy in metastatic colorectal cancer: a randomized phase III study. J Clin Oncol. 2008; 26:2013-2019.

8. Hurwitz H, Fehrenbacher L, Novotny W, et al. Bevacizumab plus irinotecan, fluorouracil, and leucovorin for metastatic colorectal cancer. N Engl J Med. 2004;350:2335-2342.

9. Zorzi D, Chun YS, Madoff DC, et al. Chemotherapy with bevacizumab does not affect liver regeneration after portal vein embolization in the treatment of colorectal liver metastases. Ann Surg Oncol. 2008;15:2765-2772.

10. Aussilhou B, Dokmak S, Faivre $S$, et al. Preoperative liver hypertrophy induced by portal flow occlusion before major hepatic resection for colorectal metastases can be impaired by bevacizumab. Ann Surg Oncol. 2009;16:1553-1559.

11. Folprecht $G$, Gruenberger $T$, Bechstein WO, et al. Tumour response and secondary resectability of colorectal liver metastases following neoadjuvant chemotherapy with cetuximab: the CELIM randomised phase 2 trial. Lancet Oncol. 2010;11:38-47.

12. Ye LC, Liu TS, Ren L, et al. Randomized controlled trial of cetuximab plus chemotherapy for patients with KRAS wild-type unresectable colorectal liver-limited metastases. J Clin Oncol. 2013;31:1931-1938.

13. Primrose J, Falk $\mathrm{S}$, Finch-Jones $\mathrm{M}$, et al. Systemic chemotherapy with or without cetuximab in patients with resectable colorectal liver metastasis: the New EPOC randomised controlled trial. Lancet Oncol. 2014;15:601-611.

14. Geissler M, Martens UM, Knorrenscheild R, et al. mFOLFOXIRI + panitumumab versus FOLFOXIRI as first-line treatment in patients with RAS wild-type metasstatic coloretcal cancer: a randomized II VOLFI trial. Ann Oncol. 2017;28(suppl 5):mdx393.

15. Guiu B, Chevallier P, Denys A, et al. Simultaneous trans-hepatic portal and hepatic vein embolization before major hepatectomy: the liver venous deprivation technique. Eur Radiol. 2016;26:4259-4267.

16. Schadde E, Tsatsaris C, Swiderska-Syn M, et al. Hypoxia of the growing liver accelerates regeneration. Surgery. 2017;161:666-679. 
17. de Baere T, Roche A, Elias D, et al. Preoperative portal vein embolization for extension of hepatectomy indications. Hepatology. 1996;24:13861391.

18. Madoff DC, Abdalla EK, Vauthey JN. Portal vein embolization in preparation for major hepatic resection: evolution of a new standard of care. J Vasc Interv Radiol. 2005;16:779-790.

19. Oussoultzoglou E, Bachellier P, Rosso E, et al. Right portal vein embolization before right hepatectomy for unilobar colorectal liver metastases reduces the intrahepatic recurrence rate. Ann Surg. 2006;244:71-79.

20. de Jong MC, van Dam RM, Maas M, et al. The liver-first approach for synchronous colorectal liver metastasis: a 5-year single-centre experience. HPB. 2011;13:745-752.

21. de Jong MC, Pulitano C, Ribero D, et al. Rates and patterns of recurrence following curative intent surgery for colorectal liver metastasis: an international multi-institutional analysis of 1669 patients. Ann Surg. 2009;250:440-448.

22. Khan NA, Baerlocher MO, Owen RJ, et al. Ablative technologies in the management of patients with primary and secondary liver cancer: an overview. Can Assoc Radiol J. 2010;61:217-222.

23. Curley SA. Radiofrequency ablation versus resection for resectable colorectal liver metastases: time for a randomized trial? Ann Surg Oncol. 2008;15:11-13.

24. Hayat MJ, Howlader N, Reichman ME, et al. Cancer statistics, trends, and multiple primary cancer analyses from the Surveillance, Epidemiology, and End Results (SEER) Program. Oncologist. 2007;12:20-37.

25. Bale R, Widmann G, Schullian P, et al. Percutaneous stereotactic radiofrequency ablation of colorectal liver metastases. Eur Radiol. 2012;22:930-937.

26. Gillams AR, Lees WR. Five-year survival in 309 patients with colorectal liver metastases treated with radiofrequency ablation. Eur Radiol. 2009;19:1206-1213.

27. Hamada A, Yamakado K, Nakatsuka A, et al. Radiofrequency ablation for colorectal liver metastases: prognostic factors in non-surgical candidates. Jpn J Radiol. 2012;30:567-574.

28. Hammill CW, Billingsley KG, Cassera MA, et al. Outcome after laparoscopic radiofrequency ablation of technically resectable colorectal liver metastases. Ann Surg Oncol. 2011;18:1947-1954.

29. Machi J, Oishi AJ, Sumida K, et al. Long-term outcome of radiofrequency ablation for unresectable liver metastases from colorectal cancer: evaluation of prognostic factors and effectiveness in first- and secondline management. Cancer J. 2006;12:318-326.

30. Ruers T, Van Coevorden F, Punt CJ, et al; European Organisation for Research and Treatment of Cancer (EORTC); Gastro-Intestinal Tract Cancer Group; Arbeitsgruppe Lebermetastasen und tumoren in der Chirurgischen Arbeitsgemeinschaft Onkologie (ALM-CAO); National Cancer Research Institute Colorectal Clinical Study Group (NCRI CCSG). Local treatment of unresectable colorectal liver metastases: results of a randomized phase II trial. J Nat/ Cancer Inst. 2017;109:109.

31. Solbiati L, Ahmed M, Cova L, et al. Small liver colorectal metastases treated with percutaneous radiofrequency ablation: local response rate and long-term survival with up to 10-year follow-up. Radiology. 2012;265:958-968.

32. Agcaoglu O, Aliyev S, Karabulut K, et al. Complementary use of resection and radiofrequency ablation for the treatment of colorectal liver metastases: an analysis of 395 patients. World J Surg. 2013;37: 1333-1339.

33. Imai K, Allard MA, Castro Benitez C, et al. Long-term outcomes of radiofrequency ablation combined with hepatectomy compared with hepatectomy alone for colorectal liver metastases. $\mathrm{Br} J$ Surg. 2017;104:570-579.

34. Kim KH, Yoon YS, Yu CS, et al. Comparative analysis of radiofrequency ablation and surgical resection for colorectal liver metastases. J Korean Surg Soc. 2011;81:25-34.

35. Van Tilborg AA, Meijerink MR, Sietses $C$, et al. Long-term results of radiofrequency ablation for unresectable colorectal liver metastases: a potentially curative intervention. Br J Radiol. 2011;84:556-565.

36. Chang DT, Swaminath A, Kozak M, et al. Stereotactic body radiotherapy for colorectal liver metastases: a pooled analysis. Cancer. 2011;117:4060-4069.

37. Salati U, Barry A, Chou FY, et al. State of the ablation nation: a review of ablative therapies for cure in the treatment of hepatocellular carcinoma. Future Oncol. 2017;13:1437-1448.

38. Liu DM, Thakor AS, Baerlocher $\mathrm{M}$, et al. A review of conventional and drug-eluting chemoembolization in the treatment of colorectal liver metastases: principles and proof. Future Oncol. 2015;11:1421-1428.

39. Martin RC II, Scoggins CR, Schreeder M, et al. Randomized controlled trial of irinotecan drug-eluting beads with simultaneous FOLFOX and bevacizumab for patients with unresectable colorectal liver-limited metastasis. Cancer. 2015;121:3649-3658.

40. Berkovic P, Gulyban A, Nguyen PV, et al. Stereotactic robotic body radiotherapy for patients with unresectable hepatic oligorecurrence. Clin Colorectal Cancer. 2017;16:349-357.e1.

41. Ahmed KA, Caudell JJ, El-Haddad G, et al. Radiosensitivity differences between liver metastases based on primary histology suggest implications for clinical outcomes after stereotactic body radiation therapy. Int J Radiat Oncol Biol Phys. 2016;95:1399-1404.

42. Swaminath $A$, Dawson LA. Emerging role of radiotherapy in the management of liver metastases. Cancer J. 2010;16:150-155.

43. Scorsetti M, Arcangeli S, Tozzi A, et al. Is stereotactic body radiation therapy an attractive option for unresectable liver metastases? A preliminary report from a phase 2 trial. Int J Radiat Oncol Biol Phys. 2013;86:336-342.

44. Tanguturi SK, Wo JY, Zhu AX, et al. Radiation therapy for liver tumors: ready for inclusion in guidelines? Oncologist. 2014;19:868-879.

45. Langen KM, Jones DT. Organ motion and its management. Int J Radiat Oncol Biol Phys. 2001;50:265-278.

46. Case RB, Sonke JJ, Moseley DJ, et al. Inter- and intrafraction variability in liver position in non-breath-hold stereotactic body radiotherapy. Int J Radiat Oncol Biol Phys. 2009;75:302-308.

47. Cho M, Kessler J, Park JJ, et al. A single institute retrospective trial of concurrent chemotherapy with SIR-Spheres versus SIR-Spheres alone in chemotherapy-resistant colorectal cancer liver metastases. J Gastrointest Oncol. 2017;8:608-613.

48. Kress MS, Collins BT, Collins SP, et al. Stereotactic body radiation therapy for liver metastases from colorectal cancer: analysis of safety, feasibility, and early outcomes. Front Oncol. 2012;2:8.

49. van der Pool AE, Méndez Romero A, Wunderink W, et al. Stereotactic body radiation therapy for colorectal liver metastases. $\mathrm{Br} J$ Surg. 2010;97:377-382. 
50. McPartlin A, Swaminath A, Wang R, et al. Long-term outcomes of phase 1 and 2 studies of SBRT for hepatic colorectal metastases. Int $J$ Radiat Oncol Biol Phys. 2017;99:388-395.

51. Blomgren H, Lax I, Näslund I, et al. Stereotactic high dose fraction radiation therapy of extracranial tumors using an accelerator. Clinical experience of the first thirty-one patients. Acta Oncol. 1995;34:861870.

52. Hoyer $\mathrm{M}$, Roed $\mathrm{H}$, Traberg Hansen $\mathrm{A}$, et al. Phase II study on stereotactic body radiotherapy of colorectal metastases. Acta Oncol. 2006;45:823830.

53. Benedict $\mathrm{SH}$, Yenice KM, Followill D, et al. Stereotactic body radiation therapy: the report of AAPM Task Group 101. Med Phys. 2010;37:40784101.

54. Lawrence TS, Robertson JM, Anscher MS, et al. Hepatic toxicity resulting from cancer treatment. Int J Radiat Oncol Biol Phys. 1995;31:1237-1248.

55. Emami B, Lyman J, Brown A, et al. Tolerance of normal tissue to therapeutic irradiation. Int J Radiat Oncol Biol Phys. 1991;21:109-122.

56. Dawson LA, Normolle D, Balter JM, et al. Analysis of radiation-induced liver disease using the Lyman NTCP model. Int J Radiat Oncol Biol Phys. 2002;53:810-821.

57. Kutcher GJ, Burman C. Calculation of complication probability factors for non-uniform normal tissue irradiation: the effective volume method. Int J Radiat Oncol Biol Phys. 1989;16:1623-1630.

58. Bae SH, Kim MS, Cho CK, et al. High dose stereotactic body radiotherapy using three fractions for colorectal oligometastases. J Surg Oncol. 2012;106:138-143.

59. Comito T, Cozzi L, Clerici E, et al. Stereotactic ablative radiotherapy (SABR) in inoperable oligometastatic disease from colorectal cancer: a safe and effective approach. BMC Cancer. 2014;14:619.

60. Kang JK, Kim MS, Kim JH, et al. Oligometastases confined one organ from colorectal cancer treated by SBRT. Clin Exp Metastasis. 2010;27:273-278.

61. Herfarth $\mathrm{KK}, \mathrm{Hof} \mathrm{H}, \mathrm{Bahner} \mathrm{ML}$, et al. Assessment of focal liver reaction by multiphasic CT after stereotactic single-dose radiotherapy of liver tumors. Int J Radiat Oncol Biol Phys. 2003;57:444-451.

62. Rusthoven KE, Kavanagh BD, Cardenes $H$, et al. Multi-institutional phase I/II trial of stereotactic body radiation therapy for liver metastases. J Clin Oncol. 2009;27:1572-1578.

63. Rule W, Timmerman R, Tong $L$, et al. Phase I dose-escalation study of stereotactic body radiotherapy in patients with hepatic metastases. Ann Surg Oncol. 2011;18:1081-1087.

64. Hijazi H, Campeau MP, Roberge D, et al. Stereotactic body radiotherapy for inoperable liver tumors: results of a single institutional experience. Cureus. 2016;8:e935.

65. Méndez Romero A, Wunderink W, Hussain SM, et al. Stereotactic body radiation therapy for primary and metastatic liver tumors: a single institution phase I-Il study. Acta Oncol. 2006;45:831-837.

66. Katz AW, Carey-Sampson M, Muhs AG, et al. Hypofractionated stereotactic body radiation therapy (SBRT) for limited hepatic metastases. Int J Radiat Oncol Biol Phys. 2007;67:793-798.

67. Wulf J, Guckenberger M, Haedinger U, et al. Stereotactic radiotherapy of primary liver cancer and hepatic metastases. Acta Oncol. 2006; 45:838-847.
68. Goodman KA, Wiegner EA, Maturen KE, et al. Dose-escalation study of single-fraction stereotactic body radiotherapy for liver malignancies. Int J Radiat Oncol Biol Phys. 2010;78:486-493.

69. Ambrosino G, Polistina F, Costantin G, et al. Image-guided robotic stereotactic radiosurgery for unresectable liver metastases: preliminary results. Anticancer Res. 2009;29:3381-3384.

70. Carrato A, Abad A, Massuti B, et al. First-line panitumumab plus FOLFOX4 or FOLFIRI in colorectal cancer with multiple or unresectable liver metastases: a randomised, phase II trial (PLANET-TTD). Eur J Cancer. 2017;81:191-202.

71. Joo JH, Park JH, Kim JC, et al. Local control outcomes using stereotactic body radiation therapy for liver metastases from colorectal cancer. Int J Radiat Oncol Biol Phys. 2017;99:876-883.

72. Doi H, Uemoto K, Suzuki O, et al. Effect of primary tumor location and tumor size on the response to radiotherapy for liver metastaes from colorectal cancer. Oncol Lett. 2017;14:453-460.

73. Kang J, Demaria S, Formenti S. Current clinical trials testing the combination of immunotherapy with radiotherapy. J Immunother Cancer. 2016;4:51.

74. Chen HH, Lin JK, Chen JB, et al. Neoadjuvant therapy of bevacizumab in combination with oxaliplatin and capecitabine (XELOX) for patients with metastatic colorectal cancer with unresectable liver metastases: a phase II, open-label, single-arm, noncomparative trial. Asia Pac J Clin Oncol. 2018;14:61-68.

75. Agolli L, Bracci S, Nicosia L, et al. Lung metastases treated with stereotactic ablative radiation therapy in oligometastatic colorectal cancer patients: outcomes and prognostic factors after long-term follow-up. Clin Colorectal Cancer. 2017;16:58-64.

76. Widder J, Klinkenberg TJ, Ubbels JF, et al. Pulmonary oligometastases: metastasectomy or stereotactic ablative radiotherapy? Radiother Oncol. 2013;107:409-413.

77. Yeung R, Hamm J, Liu M, et al. Institutional analysis of stereotactic body radiotherapy (SBRT) for oligometastatic lymph node metastases. Radiat Oncol. 2017;12:105.

78. Nicolay NH, Berry DP, Sharma RA. Liver metastases from colorectal cancer: radioembolization with systemic therapy. Nat Rev Clin Oncol. 2009;6:687-697.

79. Kennedy A, Nag S, Salem R, et al. Recommendations for radioembolization of hepatic malignancies using yttrium-90 microsphere brachytherapy: a consensus panel report from the radioembolization brachytherapy oncology consortium. Int J Radiat Oncol Biol Phys. 2007;68:13-23.

80. Morgan B, Kennedy AS, Lewington V, et al. Intra-arterial brachytherapy of hepatic malignancies: watch the flow. Nat Rev Clin Oncol. 2011;8: 115-120.

81. Mulcahy MF, Lewandowski RJ, Ibrahim SM, et al. Radioembolization of colorectal hepatic metastases using yttrium-90 microspheres. Cancer. 2009;115:1849-1858.

82. Seidensticker R, Denecke T, Kraus $P$, et al. Matched-pair comparison of radioembolization plus best supportive care versus best supportive care alone for chemotherapy refractory liver-dominant colorectal metastases. Cardiovasc Intervent Radiol. 2012;35:1066-1073.

83. Hendlisz A, Van den Eynde M, Peeters M, et al. Phase III trial comparing protracted intravenous fluorouracil infusion alone or with yttrium-90 resin microspheres radioembolization for liver-limited metastatic colorectal cancer refractory to standard chemotherapy. J Clin Oncol. 2010;28:3687-3694. 
84. van Hazel GA, Pavlakis N, Goldstein D, et al. Treatment of fluorouracilrefractory patients with liver metastases from colorectal cancer by using yttrium-90 resin microspheres plus concomitant systemic irinotecan chemotherapy. J Clin Oncol. 2009;27:4089-4095.

85. Pardo F, Sangro B, Lee RC, et al. The Post-SIR-Spheres Surgery Study (P4S): retrospective analysis of safety following hepatic resection or transplantation in patients previously treated with selective internal radiation therapy with yttrium-90 resin microspheres. Ann Surg Oncol. 2017;24:2465-2473.
86. Sharma RA, Wasan HS, Love SB, et al. FOXFIRE: a phase III clinical trial of chemo-radio-embolisation as first-line treatment of liver metastases in patients with colorectal cancer. Clin Oncol. 2008;20:261-263.

87. Wasan HS, Gibbs P, Sharma NK, et al; FOXFIRE trial investigators; SIRFLOX trial investigators; FOXFIRE-Global trial investigators. First-line selective internal radiotherapy plus chemotherapy versus chemotherapy alone in patients with liver metastases from colorectal cancer (FOXFIRE, SIRFLOX, and FOXFIRE-Global): a combined analysis of three multicentre, randomised, phase 3 trials. Lancet Oncol. 2017;18:1159-1171. 